\title{
Cost-Effectiveness of Rifaximin Treatment in Patients with Hepatic Encephalopathy
}

\author{
Arun B. Jesudian, MD; Maliha Ahmad, MD; Duygu Bozkaya, MSc, MBA;
} and Kristen Migliaccio-Walle, BS

\begin{abstract}
BACKGROUND: Hepatic encephalopathy (HE) is a complication of cirrhosis of the liver causing neuropsychiatric abnormalities. Clinical manifestations of overt $\mathrm{HE}$ result in increased health care resource utilization and effects on patient quality of life. While lactulose has historically been the mainstay of treatment for acute HE and maintenance of remission, there is an unmet need for additional therapeutic options with a favorable adverse event profile. Compared with lactulose alone, rifaximin has demonstrated proven efficacy in complete reversal of $\mathrm{HE}$ and reduction in the incidence of $\mathrm{HE}$ recurrence, mortality, and hospitalizations. Evidence suggests the benefit of long-term prophylactic therapy with rifaximin; however, there is a need to assess the economic impact of rifaximin treatment in patients with HE.
\end{abstract}

OBJECTIVE: To assess the incremental cost-effectiveness of rifaximin \pm lactulose versus lactulose monotherapy in patients with overt $\mathrm{HE}$.

METHODS: A Markov model was developed in Excel with 4 health states (remission, overt HE, liver transplantation, and death) to predict costs and outcomes of patients with HE after initiation of maintenance therapy with rifaximin \pm lactulose to avoid recurrent $\mathrm{HE}$ episodes. Cost-effectiveness of rifaximin was evaluated through estimation of incremental cost per qualityadjusted life-year (QALY) or life-year (LY) gained. Analyses were conducted over a lifetime horizon. One-way deterministic and probabilistic sensitivity analyses were conducted to assess uncertainty in results.

RESULTS: The rifaximin \pm lactulose regimen provided added health benefits despite an additional cost versus lactulose monotherapy. Model results showed an incremental benefit of $\$ 29,161$ per QALY gained and $\$ 27,762$ per LY gained with rifaximin \pm lactulose versus lactulose monotherapy. Probabilistic sensitivity analyses demonstrated that the rifaximin \pm lactulose regimen was cost-effective $\sim 99 \%$ of the time at a threshold of $\$ 50,000$ per QALY/LY gained, which falls within the commonly accepted threshold for incremental cost-effectiveness.

CONCLUSIONS: The clinical benefit of rifaximin, combined with an acceptable economic profile, demonstrates the advantages of rifaximin maintenance therapy as an important option to consider for patients at risk of recurrent $\mathrm{HE}$.

J Manag Care Spec Pharm. 2020;26(6):750-57

Copyright $\odot 2020$, Academy of Managed Care Pharmacy. All rights reserved.

\section{What is already known about this subject}

Hepatic encephalopathy (HE) is a complication of significant liver disease that causes neuropsychiatric abnormalities and results in increased health care resource utilization

Lactulose has historically been the first-line treatment for acute episodes of HE and maintenance of remission but is limited by inadequate efficacy and safety.

Rifaximin + lactulose combination therapy has demonstrated greater benefit in reducing HE recurrence and length of hospital stay compared with lactulose alone.

\section{What this study adds}

This research addresses limitations from a previous economic analysis of rifaximin for the treatment of patients with HE.

The current analysis demonstrates that the clinical benefits of a rifaximin-based regimen may be obtained at reasonable cost to

U.S. payers relative to lactulose monotherapy.

$\mathrm{H}$ epatic encephalopathy (HE) is a complication of cirrhosis of the liver causing neuropsychiatric abnormalities. ${ }^{1}$

Approximately $30 \%-45 \%$ of patients with cirrhosis and $10 \%-50 \%$ of patients who require transjugular intrahepatic portosystemic shunt (TIPS) insertion for complications of liver disease experience episodes of overt HE..$^{2-4}$ The establishment of a TIPS bypasses ammonia and other neurotoxins directly into systemic circulation, increasing the incidence of HE, which often occurs soon after (i.e., within 3 months) TIPS insertion; over time, there is cerebral adaptation to gut-derived neurotoxins. A major risk factor for post-TIPS development of HE is a previous history of HE. ${ }^{5}$ Clinical manifestations of overt HE include generalized psychomotor dysfunction with alterations in consciousness. ${ }^{6}$ Episodes of overt HE may result in increased health care resource utilization and can affect quality of life of both patients and families. ${ }^{7,8}$

After an initial episode of overt HE, secondary prophylaxis is recommended to decrease the risk of recurrence, which is considered preventable with proper prophylaxis. ${ }^{9-12}$ Lactulose has historically been used as first-line therapy for acute HE and for maintenance of remission..$^{6,13,14}$ Use of lactulose as a maintenance medication may be limited because of a lack of randomized, placebo-controlled trials to determine efficacy. ${ }^{14,15}$ 


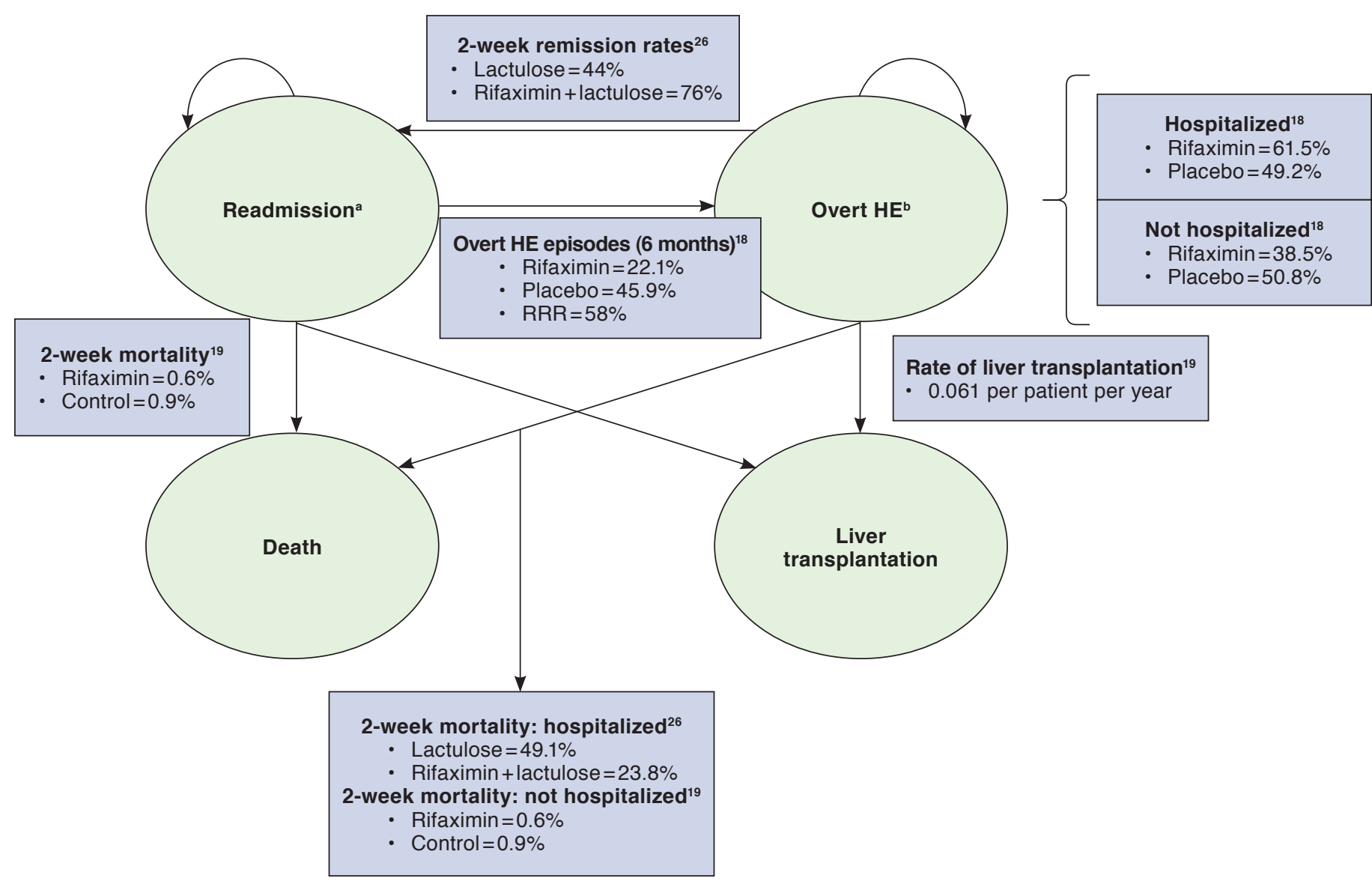

a At risk of hospitalizations that were not HE-related.

${ }^{b}$ At risk of HE-related hospitalizations.

$H E=$ hepatic encephalopathy; $R R R=$ relative risk reduction

In addition, lactulose is associated with unpleasant or sweet taste, dehydration, and gastrointestinal adverse events (AEs), such as diarrhea, nausea, bloating, and gas, ${ }^{6,14}$ which may lead to medication nonadherence. ${ }^{16}$ Given these concerns, additional HE therapies with a more favorable AE profile have been investigated in the treatment of overt HE.

Rifaximin (Xifaxan $550 \mathrm{mg}$ tablets) is a minimally absorbed, oral antimicrobial agent that was approved for reduction in the risk of overt HE recurrence in adults by the U.S. Food and Drug Administration in 2010. ${ }^{17}$ This therapy is often used concomitantly with lactulose. ${ }^{15}$ In a randomized controlled trial over a 6-month period, rifaximin maintained remission from HE and reduced the risk of HE-related hospitalizations more effectively than placebo in a cohort of patient with decompensated cirrhosis and previous episodes of overt HE, with approximately $90 \%$ in both arms receiving concomitant lactulose. ${ }^{18}$ The rates of AEs and hospitalizations were similar in a 24-month openlabel follow-up study. ${ }^{19}$

Additional research indicates that rifaximin may be beneficial in reducing incidence of $\mathrm{HE}$ recurrence, ${ }^{20-24}$ mortality, ${ }^{20,21,23,24}$ and rate of hospitalizations by up to $58 \%,{ }^{21,22}$ which may translate to a decrease in the health care cost burden attributed to HE.

This study aimed to assess the incremental cost-effectiveness of rifaximin \pm lactulose versus lactulose monotherapy in patients with overt HE. For this purpose, a cost-effectiveness model of patients who were in remission from recurrent HE was developed.

\section{Methods}

Using Microsoft Excel, a cost-effectiveness model was created to predict costs and outcomes of patients with HE after 


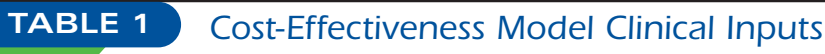

\begin{tabular}{|c|c|c|c|}
\hline Input & Rifaximin \pm Lactulose & Placebo \pm Lactulose & Source \\
\hline \multicolumn{4}{|l|}{ Population inputs } \\
\hline On concomitant lactulose, $\%$ & 91.4 & 91.2 & Bass $2010^{18}$ \\
\hline \multicolumn{4}{|l|}{ Remission state inputs } \\
\hline With overt episodes by 6 months, $\%^{a}$ & 22.1 & 45.9 & Bass $2010^{18}$ \\
\hline Hospitalizations per person-years of exposure & $0.24 \mathrm{~b}$ & $0.58^{\mathrm{c}}$ & Mullen 201419 \\
\hline Mortality at year $5, \% \mathrm{~d}$ & 52.8 & 69.9 & Mullen 201419 \\
\hline Health utility & \multicolumn{2}{|c|}{$0.937 \mathrm{e}$} & Guest 201428 \\
\hline \multicolumn{4}{|l|}{ Overt state inputs } \\
\hline Hospitalized among those with an overt episode, \% & 61.5 & 49.2 & Bass $2010^{18}$ \\
\hline Reversed after 2 weeks among hospitalized patients, \% & 76.0 & 44.0 & Sharma 201326 \\
\hline In-hospital 2-week mortality, \% & 23.8 & 49.1 & Sharma 201326 \\
\hline Two-week mortality after hospitalization, \%d & 0.6 & 0.9 & Mullen 201419 \\
\hline Nonhospitalized 2-week mortality, \% $\mathrm{d}$ & 0.6 & 0.9 & Mullen 201419 \\
\hline Health utility for HE & \multicolumn{2}{|c|}{$0.783^{f}$} & Bass 201018; Guest $2014^{28}$ \\
\hline \multicolumn{4}{|l|}{ Liver transplantation inputs } \\
\hline Number of liver transplantations per patient per year & 0.061 & 0.061 & Mullen 201419 \\
\hline Life expectancy after liver transplantationg & \multicolumn{2}{|c|}{29.3 years } & $\begin{array}{l}\text { OPTN and SRTR } 2016 \\
\text { Annual Data Report } \\
\text { (Kim 201827) }\end{array}$ \\
\hline Health utility after liver transplantation & \multicolumn{2}{|c|}{$0.962 \mathrm{~h}$} & Guest 201428 \\
\hline \multicolumn{4}{|c|}{$\begin{array}{l}\text { aTime to first breakthrough HE episode Kaplan-Meier curves from Bass } 2010^{18} \text { were fit to follow Weibull distributions with } \gamma \text { and } \lambda \text { parameters. The } \gamma \text { parameter is } f \\
\text { in the analysis, and percentage at } 6 \text { months from Bass } 2010^{18} \text { is used to calibrate the } \lambda \text { parameter (rifaximin group } \gamma=0.6376, \lambda=0.2466 ; \text { lactulose group } \gamma=0.7716 \text {, } \\
\lambda=1.1555 \text { ). } \\
\text { bBased on all-rifaximin group. } \\
\text { 'Based on historical placebo group. } \\
{ }^{d} \text { Constant hazard assumed based on events per person-years of exposure. } \\
\text { eAverage of reported health utilities for Conn grades } 0 \text { and } 1 . \\
\text { fWeighted average of health utilities reported for Conn grades 2, 3, and } 428 \text { was taken based on distribution of Conn scores during most recent HE episode before the } \\
\text { rifaximin study. } 18\end{array}$} \\
\hline
\end{tabular}

initiation of maintenance therapy with rifaximin \pm lactulose to avoid recurrent $\mathrm{HE}$ episodes. Rifaximin \pm lactulose refers to the use of rifaximin $550 \mathrm{mg}$ twice daily \pm lactulose, reflecting a population consistent with the rifaximin phase 3 maintenance of remission trial in which $91.4 \%$ of patients received both medications. ${ }^{18}$

Poor compliance and adherence to HE therapy contributes to increased disease burden. ${ }^{25}$ Because of a lack of available data to explicitly model these factors, the potential effect of poor compliance with, or adherence to, therapy was captured indirectly via observed risks of overt HE. Quality-adjusted life-years (QALYs) and life-years (LY) were evaluated for both cohorts in the context of the direct costs associated with each treatment regimen. The analysis was conducted from a thirdparty U.S. payer perspective.

\section{Model Structure}

A Markov model was used to predict the course of HE following initiation of maintenance therapy to avoid recurrent HE episodes. Patients are assumed to begin in the remission state, where they are at risk for an overt HE episode with or without hospitalization, death, or liver transplant in each 2-week cycle over a lifetime horizon (Figure 1). Patients in the overt HE state (with or without a hospitalization) can transition back to the remission state, die, or receive a liver transplant. Patients transitioning to the death state exit the model after accruing appropriate costs and outcomes. The liver transplantation state is also an absorbing or exit state. Patients accrue the cost of transplantation, the average life expectancy after transplantation is applied, and QALYs/LYs are accrued.

\section{Clinical Inputs}

Clinical inputs and data sources for the model are provided in Table 1. The risk of an initial HE episode was based on clinical trial data. ${ }^{18}$ The risk of a subsequent episode of overt HE was based on the initial 2-week hazard function estimate and was assumed to be invariable over time. The effect of varying risk (excess risk with each additional episode) was tested in the 
TABLE 2 Cost-Effectiveness Model Cost Inputs

\begin{tabular}{|c|c|c|c|}
\hline sts & & & \\
\hline st of HE-related hospitalization ${ }^{b}$ & & & Leevy 200730; BLS 201833; CMS FY 2 \\
\hline st & \multicolumn{2}{|c|}{15,892} & 17 \\
\hline ost of liver transplantation d,21,23,25 & \multicolumn{2}{|c|}{183,132} & Wai 201432; BLS $2018^{3}$ \\
\hline \multicolumn{4}{|r|}{$\begin{array}{l}\text { care component of Consumer Price Index 2018,33 cost-to-charge } \\
\text { 67.23, 456.0) inflated to } 2018 \text { cost and converted to private } \\
\text { are component of Consumer Price Index } 33 \text { and } 2016 \text { payment- } \\
\text { of Labor Statistics; CMS = Centers for Medicare E Medicaid } \\
\text { CD-9-CM = International Classification of Diseases, Ninth }\end{array}$} \\
\hline
\end{tabular}

sensitivity analysis, since this excess risk is not reported in the literature.

Upon transition to an overt HE episode, the proportion of patients requiring an HE-related hospitalization was applied to the rifaximin +lactulose alone patients separately using estimates from the literature. ${ }^{18}$ Patients with an overt episode requiring hospitalization remained in the overt state until remission, death, or liver transplantation. Rates of reversal for patients hospitalized with overt HE and initial 2-week inpatient mortality were obtained from a randomized controlled trial comparing treatment with a rifaximin regimen with lactulose alone. ${ }^{26}$ Mortality after discharge was estimated from a 24-month open-label study of rifaximin from which biweekly rates were calculated and applied. ${ }^{19}$ For these patients, the average cost of a hospitalization was applied once during the cycle corresponding to the time an overt episode occurred.

The remaining proportion of patients transitioning to overt HE was assumed not to be hospitalized. Mortality during this period was assumed to be the same as the postdischarge mortality for hospitalized patients. ${ }^{19}$ All nonhospitalized patients were assumed to recover within 2 weeks and revert completely to the remission state.

Rates of liver transplantation were assumed to be equal for patients receiving rifaximin \pm lactulose, regardless of $\mathrm{HE}$ status. Probability of transplantation was obtained from the open-label rifaximin study, ${ }^{19}$ and survival among transplant recipients was adjusted according to published life expectancy estimates. $^{27}$

\section{Health Utility Inputs}

All health utility values for patients with overt $\operatorname{HE~(0.783),~}$ patients with decompensated cirrhosis in remission/no overt HE (0.937), and patients receiving liver transplant (0.962) were obtained from published literature..$^{18,28}$

\section{Costs}

Wholesale drug acquisition costs, hospitalization costs, and costs related to liver transplant were included in the model (Table 2). ${ }^{29-32}$ The full cost of therapy was included in the model, since there were insufficient data to adjust for compliance and adherence in the base analysis. Indirect costs were not included because the analysis was from the U.S. payer perspective. Costs were estimated and reported in 2018 U.S. dollars. If charges from previous years were used, they were inflated to current values using the medical care component of the Consumer Price Index and adjusted to private payer payments. ${ }^{33-35}$

\section{Analysis}

Total costs associated with each treatment were reported in aggregate and by component (drug, other direct, hospitalization, and liver transplantation costs). LYs, QALYs, and costs were discounted at an annual rate of 3.0\%. Cost-effectiveness of rifaximin was assessed through estimation of the incremental cost per QALY gained and the cost per LY gained. Analyses were conducted over a lifetime horizon. Threshold analyses were conducted to estimate the wholesale acquisition cost (WAC) at which rifaximin would need to be set to achieve a cost per QALY of $\$ 50,000, \$ 100,000$, and $\$ 150,000$.

One-way deterministic sensitivity analyses were conducted on individual parameters to estimate the potential impact of variability in these estimates defined by the boundaries of the standard error for each input (Table 3). Probabilistic sensitivity analyses, where all parameters are varied simultaneously in a simulation by randomly sampling values based on predefined distributions (Table 3), ${ }^{36}$ were also conducted to estimate the robustness of the results over the range of potential input values defined by the standard error and shape of the distribution. 


\begin{tabular}{|c|c|c|c|}
\hline \multirow[b]{2}{*}{ Input } & \multicolumn{2}{|c|}{$\begin{array}{c}\text { Range of Inputs Used for Deterministic and Probabilistic } \\
\text { Sensitivity Analyses }\end{array}$} & \multirow{2}{*}{$\begin{array}{c}\text { Probabilistic Sensitivity } \\
\text { Analysis Statistical } \\
\text { Distribution }\end{array}$} \\
\hline & Rifaximin \pm Lactulose & Placebo \pm Lactulose & \\
\hline \multicolumn{4}{|l|}{ Population } \\
\hline On concomitant lactulose, $\%$ & $86.2-95.5$ & $86.3-95.1$ & Beta \\
\hline \multicolumn{4}{|l|}{ Remission state } \\
\hline With overt episodes by 6 months, $\%^{a}$ & $15.2-29.0$ & $38.2-53.6$ & Normal \\
\hline Hospitalizations per person-years exposure & $0.18-0.30$ & $0.44-0.73$ & Gamma \\
\hline Mortality at year $5, \%^{a}$ & $47.9-58.0$ & $62.8-77.0$ & Normal \\
\hline Health utility & \multicolumn{2}{|c|}{$0.92-0.95$} & Beta \\
\hline \multicolumn{4}{|l|}{ Overt state } \\
\hline Hospitalization among those with an overt episode, \% & $53.3-69.4$ & $41.4-57.0$ & Beta \\
\hline Reversed after 2 weeks among hospitalized patients, $\%$ & $64.7-85.7$ & $31.4-57.0$ & Beta \\
\hline In-hospital 2-week mortality, \% & $14.1-35.1$ & $36.2-62.1$ & Beta \\
\hline Two-week mortality after hospitalization, \% & $0.1-1.6$ & $0.1-2.8$ & Beta \\
\hline Nonhospitalized 2-week mortality, \% & $0.1-1.6$ & $0.1-2.8$ & Beta \\
\hline Health utility for HE & \multicolumn{2}{|c|}{$0.74-0.82$} & Beta \\
\hline \multicolumn{4}{|l|}{ Liver transplantation } \\
\hline Number of liver transplantations per patient per year & \multicolumn{2}{|c|}{$0.05-0.08$} & Gamma \\
\hline Life expectancy after liver transplantation & \multicolumn{2}{|c|}{$24.0-35.0$ years } & Normal \\
\hline Health utility after liver transplantation & \multicolumn{2}{|c|}{$0.95-0.97$} & Beta \\
\hline \multicolumn{4}{|l|}{ Costs, \$ } \\
\hline Lactulose daily cost & \multicolumn{2}{|c|}{$1.16-1.45$} & Gamma \\
\hline Cost of HE-related hospitalization & $15,093-24,934$ & $18,782-31,026$ & Gamma \\
\hline Cost of non-HE-related hospitalization & \multicolumn{2}{|c|}{$12,169-20,104$} & Gamma \\
\hline Cost of liver transplantation & \multicolumn{2}{|c|}{$169,574-197,204$} & Gamma \\
\hline \multicolumn{4}{|l|}{ Other variables } \\
\hline Relative risk for subsequent overt episodes ${ }^{b}$ & \multicolumn{2}{|c|}{$0.25-2.00$} & Lognormal \\
\hline Discount rate, costs, $\%$ & \multicolumn{2}{|c|}{$0.0-6.0$} & N/A \\
\hline Discount rate, benefits, $\%$ & \multicolumn{2}{|c|}{$0.0-6.0$} & N/A \\
\hline \multicolumn{4}{|c|}{$\begin{array}{l}\text { Note: Values shown in this table are standard error ranges for default parameters shown in Tables } 1 \text { and } 2 . \\
\text { a Hazard parameter varied in probabilistic sensitivity analysis. } \\
\text { bBase case value is } 1 . \\
\text { HE= hepatic encephalopathy; N/A= not applicable. }\end{array}$} \\
\hline
\end{tabular}

\section{Results}

Predicted QALYs and LYs associated with rifaximin \pm lactulose were twice as high compared with lactulose alone (Table 4). Because of this extended life expectancy, the number of patients receiving liver transplantation doubled with rifaximin \pm lactulose, with an estimated 20 patients predicted to reach transplantation compared to 9 with lactulose alone (Table 4). The added health benefits with rifaximin \pm lactulose were achieved at an additional cost of $\$ 96,375$ over lactulose alone (Table 4), with an incremental cost per QALY gained of \$29,161 and the incremental cost per LY gained of $\$ 27,762$.

The cost-effectiveness of a rifaximin \pm lactulose regimen remained between $\sim \$ 20,000$ per QALY and $\sim \$ 40,000$ per QALY across univariate sensitivity analyses, irrespective of the parameter considered. At the time the model was developed, the WAC for rifaximin was $42 \%$ below the cost to exceed the
$\$ 50,000$ per QALY threshold and $71 \%$ and $81 \%$ below the cost to exceed the $\$ 100,000$ per QALY and $\$ 150,000$ per QALY thresholds, respectively. Outcomes were most sensitive to variation in the relative risk of subsequent overt $\mathrm{HE}$ episodes with lactulose monotherapy. Outcomes were most sensitive to variation in the relative risk of subsequent overt HE episodes with lactulose monotherapy.

In probabilistic sensitivity analyses, when multiple parameters were varied simultaneously, the combination of rifaximin \pm lactulose was estimated to be cost-effective over half the time at a willingness-to-pay threshold of $\sim \$ 28,000$ per QALY/ LY gained or higher. This regimen was predicted to be costeffective $~ 99 \%$ of the time at a threshold of $\$ 50,000$ per QALY/ LY gained. These estimates are within the commonly accepted threshold for incremental cost-effectiveness of $\$ 50,000 .{ }^{37}$ 


\section{TABLE 4 Clinical and Economic Outcomes}

\begin{tabular}{|c|c|c|c|}
\hline Outcome & Rifaximin + Lactulose & Placebo + Lactulose & Difference \\
\hline \multicolumn{4}{|l|}{ Clinical outcomes } \\
\hline QALYs per patient, discounted (undiscounted) & $6.4(8.9)$ & $3.1(4.1)$ & $3.3(4.9)$ \\
\hline LYs per patient, discounted (undiscounted) & $6.7(9.4)$ & $3.2(4.3)$ & $3.5(5.1)$ \\
\hline Number of liver transplantations (per 100) & 20 & 9 & 11 \\
\hline \multicolumn{4}{|l|}{ Costs, $\$$} \\
\hline Drug costs & 84,521 & 664 & 83,857 \\
\hline Other direct costs & 67,885 & 55,367 & 12,518 \\
\hline Hospitalizations & 34,036 & 39,316 & $-5,280$ \\
\hline HE-related & 22,469 & 26,257 & $-3,789$ \\
\hline Non-HE-related & 11,568 & 13,059 & $-1,491$ \\
\hline Liver transplantation & 33,849 & 16,051 & 17,798 \\
\hline Total & 152,406 & 56,031 & 96,375 \\
\hline
\end{tabular}

\section{Discussion}

Current guidelines recommend prophylactic treatment following an episode of overt HE to reduce the risk of recurrent HE. ${ }^{15}$ Considerable evidence suggests the benefit of long-term prophylactic therapy with rifaximin ${ }^{1,19-21,23,24,26,38,39}$; however, evidence evaluating the economic effect of rifaximin treatment is lacking. The few published resource utilization studies identified consider the costs associated with HE in general, ${ }^{25}$ in the acute inpatient setting, ${ }^{8}$ or in diagnostic screening in patients with minimal HE. ${ }^{39}$

A study by Huang et al. (2007) concluded that although rifaximin monotherapy is clinically safe and effective, ${ }^{40}$ when used as monotherapy, it is unlikely to be cost-effective under the majority of circumstances. Alternatively, a recent systematic review of studies reporting economic data for HE and rifaximin and/or lactulose found that rifaximin had a favorable economic profile. ${ }^{41}$ The current analysis demonstrates that the clinical benefits of a rifaximin-based regimen may be obtained at reasonable cost to U.S. payers relative to lactulose monotherapy and that the price of rifaximin provides good value relative to commonly accepted cost per QALY thresholds.

The present study improves on the identified limitations of the Huang et al. economic analysis, ${ }^{40}$ which served as a basis for this analysis. Differences in the original economic analysis and the present study may account for differences in overall findings. Studies were conducted over a decade apart, during which time significant contributions to the knowledge and understanding of rifaximin treatment for overt HE have been made. ${ }^{18-20,23,24,26,38,42}$ This economic analysis was able to include data from head-to-head comparison studies of rifaximin \pm lactulose, ${ }^{18}$ including long-term follow-up data. ${ }^{19}$ The Huang et al. paper relied on studies of varying duration and design to inform the analysis. ${ }^{40}$ The current analysis also includes a rifaximin + lactulose treatment arm not considered in the 2007 Huang study, partially due to the refinement of treatment guidelines for HE after its publication. ${ }^{15,40}$ Finally, understanding of the potential effect of HE on patient-centered outcomes (e.g., quality of life) has evolved during the time period between these 2 economic analyses. ${ }^{28}$

\section{Limitations}

There are potential assumptions and limitations that must be considered when interpreting the results of this model. Simplifying assumptions were made to account for data shortcomings, with a goal of achieving balance between face validity of the model and the intricacies of clinical practice and disease progression. For example, the model did not explicitly take into account patient compliance to therapy because the available data were considered to be inadequate. The treatment effectiveness inputs should inherently reflect the underlying compliance of patients within the context of the included studies. Costs of drugs assumed full compliance, which should result in conservative estimates of the overall benefit of a rifaximin regimen by allocating the full cost of therapy whether or not a patient achieves the full clinical benefit of therapy.

While it is theoretically possible that HE treatment may have a disease-modifying effect (i.e., reversal of decompensation), the current model did not account for this potential. Therefore, the model may underestimate the actual benefits of long-term maintenance therapy with rifaximin.

In addition, patients still in the overt HE state at 2 weeks after hospital discharge were assumed to have the same risk of death as patients with overt HE who were not hospitalized. Estimates were based on observed deaths in an open-label extension study under the assumption of a constant hazard function. ${ }^{19}$ It may be assumed that patients who are hospitalized for an overt HE episode are more severe or at higher risk of death than those managed in the outpatient setting.

Patients may also be at risk of ongoing neurological complications following recurrent episodes after transplant. ${ }^{43-45}$ These 
aspects are captured in the elevated mortality risk during hospitalization. Risk of subsequent overt HE episodes was calculated based on the initial hazard function estimate. Variation in these parameters did not substantially affect the results.

Finally, health utility estimates used in the model were obtained from a study using the standard gamble and time trade-off techniques, ${ }^{28}$ and the estimates appear to be lower than what has previously been published for HE. ${ }^{46}$ Hence, a separate scenario where overt and remission state utilities were taken as 0.55 and 0.74 , respectively, was implemented based on a survey of 114 patients with cirrhosis. ${ }^{46}$ This resulted in a slight increase in incremental cost-effectiveness of $\$ 32,182$ per QALY gained for rifaximin.

\section{Conclusions}

This model expands on the work of Huang et al. by integrating current clinical evidence and guidelines. ${ }^{40}$ The clinical benefit of rifaximin, combined with an acceptable economic profile, demonstrates the advantages of rifaximin maintenance therapy as an important option to consider for patients at risk of recurrent HE.

\section{Authors}

ARUN B. JESUDIAN, MD, Weill Cornell Medicine, New York, New York, and MALIHA AHMAD, MD, St. Joseph's Health, Paterson, New Jersey. DUYGU BOZKAYA, MSc, MBA, and KRISTEN MIGLIACCIO-WALLE, BS, Xcenda, Palm Harbor, Florida.

AUTHOR CORRESPONDENCE: Kristen Migliaccio-Walle, BS, Xcenda, 4114 Woodlands Pkwy, Ste. 500, Palm Harbor, FL 34685.

Tel.: 603.521.2000; E-mail: Kristen.Migliaccio@xcenda.com.

\section{DISCLOSURES}

This analysis was funded by Salix Pharmaceuticals, a division of Bausch Health US. Salix and Xcenda collaborated on the methods, and Salix, Xcenda, Jesudian, and Ahmad collaborated on the writing of the manuscript and interpretation of results. Bozkaya and Migliaccio-Walle are employees of Xcenda. Ahmad reports speaker fees from Salix Pharmaceuticals, unrelated to this study. Jesudian reports consulting and speaker fees from Salix Pharmaceuticals, unrelated to this study.

The results from this model were presented at AASLD: The Liver Meeting 2014; November 7-11; Boston, MA.

\section{ACKNOWLEDGMENTS}

The authors acknowledge Minha Choi, PharmD, and Kylie Matthews, of Xcenda, Palm Harbor, FL, for medical writing and editorial support, which was funded by Salix Pharmaceuticals. The authors also acknowledge Howard Franklin, MD, MBA, an employee of Salix, for his clinical review of the manuscript.

\section{REFERENCES}

1. Bajaj JS, Wade JB, Sanyal AJ. Spectrum of neurocognitive impairment in cirrhosis: implications for the assessment of hepatic encephalopathy. Hepatology. 2009;50(6):2014-21.

2. Poordad FF. Review article: the burden of hepatic encephalopathy. Aliment Pharmacol Ther. 2007;25(Suppl 1):3-9.

3. Elwir S, Rahimi RS. Hepatic encephalopathy: an update on the pathophysiology and therapeutic options. J Clin Transl Hepatol. 2017;5(2):142-51.

4. Munoz SJ. Hepatic encephalopathy. Med Clin North Am. 2008;92(4):795812, viii.

5. Copelan A, Kapoor B, Sands M. Transjugular intrahepatic portosystemic shunt: indications, contraindications, and patient work-up. Semin Intervent Radiol. 2014;31(3):235-42.

6. Chacko KR, Sigal SH. Update on management of patients with overt hepatic encephalopathy. Hosp Pract (1995). 2013;41(3):48-59.

7. Bajaj JS, Wade JB, Gibson DP, et al. The multi-dimensional burden of cirrhosis and hepatic encephalopathy on patients and caregivers. Am J Gastroenterol. 2011;106(9):1646-53.

8. Stepanova M, Mishra A, Venkatesan C, Younossi ZM. In-hospital mortality and economic burden associated with hepatic encephalopathy in the United States from 2005 to 2009. Clin Gastroenterol Hepatol. 2012;10(9): 1034-4l.el.

9. Acharya C, Bajaj JS. Current management of hepatic encephalopathy. Am J Gastroenterol. 2018;113(11):1600-12

10. Kornerup LS, Gluud LL, Vilstrup H, Dam G. Update on the therapeutic management of hepatic encephalopathy. Curr Gastroenterol Rep. 2018;20(5):21.

11. Saab S. Evaluation of the impact of rehospitalization in the management of hepatic encephalopathy. Int J Gen Med. 2015;8:165-73.

12. Volk ML, Tocco RS, Bazick J, Rakoski MO, Lok AS. Hospital readmissions among patients with decompensated cirrhosis. Am J Gastroenterol. 2012;107(2):247-52

13. Blei AT, Cordoba J, Practice Parameters Committee of the American College of Gastroenterology. Hepatic encephalopathy. Am J Gastroenterol. 2001;96(7):1968-76.

14. Thompson JR. Treatment guidelines for hepatic encephalopathy. Pharmacotherapy. 2010;30(5 Pt 2):4S-9S.

15. Vilstrup H, Amodio P, Bajaj J, et al. Hepatic encephalopathy in chronic liver disease: 2014 Practice Guideline by the American Association for the Study of Liver Diseases and the European Association for the Study of the Liver. Hepatology. 2014;60(2):715-35.

16. Bajaj J, Sanyal A, Bell D, Gilles H, Hueuman D. Predictors of the recurrence of hepatic encephalopathy in lactulose-treated patients. Aliment Pharmacol Ther. 2010;31(9):1012-17.

17. Xifaxan (rifaximin) tablets, for oral use. Salix Pharmaceuticals. January 2019. Available at: https://shared.salix.com/shared/pi/xifaxan550-pi.pdf. Accessed April 28, 2020.

18. Bass NM, Mullen KD, Sanyal A, et al. Rifaximin treatment in hepatic encephalopathy. N Engl J Med. 2010;362(12):1071-81.

19. Mullen KD, Sanyal AJ, Bass NM, et al. Rifaximin is safe and well tolerated for long-term maintenance of remission from overt hepatic encephalopathy. Clin Gastroenterol Hepatol. 2014;12(8):1390-97.e2.

20. Bannister CA, Orr JG, Reynolds AV, et al. Natural history of patients taking rifaximin-alpha for recurrent hepatic encephalopathy and risk of future overt episodes and mortality: a post-hoc analysis of clinical trials data. Clin Ther. 2016;38(5):1081-89.e4.

21. Hudson M, Schuchmann M. Long-term management of hepatic encephalopathy with lactulose and/or rifaximin: a review of the evidence. Eur J Gastroenterol Hepatol. 2019;31(4):434-50. 
22. Bajaj JS, Barrett AC, Bortey E, Paterson C, Forbes WP. Prolonged remission from hepatic encephalopathy with rifaximin: results of a placebo crossover analysis. Aliment Pharmacol Ther. 2015;41(1):39-45.

23. Kang SH, Lee YB, Lee JH, et al. Rifaximin treatment is associated with reduced risk of cirrhotic complications and prolonged overall survival in patients experiencing hepatic encephalopathy. Aliment Pharmacol Ther. 2017:46(9):845-55.

24. Ryan JD, Tsochatzis EA. Rifaximin treatment for encephalopathy reduces hospital resource use: real-world data don't fail to IMPRESS. Frontline Gastroenterol. 2017;8(4):230-31.

25. Neff G. Factors affecting compliance and persistence with treatment for hepatic encephalopathy. Pharmacotherapy. 2010;30(5 Pt 2):22S-27S.

26. Sharma BC, Sharma P, Lunia MK, Srivastava S, Goyal R, Sarin SK. A randomized, double-blind, controlled trial comparing rifaximin plus lactulose with lactulose alone in treatment of overt hepatic encephalopathy. Am J Gastroenterol. 2013;108(9):1458-63.

27. Kim WR, Lake JR, Smith JM, et al. OPTN/SRTR 2016 annual data report: Liver. Am J Transplantation. 2018;18(S1):172-253.

28. Guest JF, Nanuwa K, Barden R. Utility values for specific hepatic encephalopathy health states elicited from the general public in the United Kingdom. Health Qual Life Outcomes. 2014;12:89.

29. IBM Watson Health. IBM Micromedex RED BOOK. [Database]. 2018. Available at: https://www.micromedexsolutions.com/micromedex2/librarian/ ssl/true. Accessed April 28, 2020.

30. Leevy CB, Phillips JA. Hospitalizations during the use of rifaximin versus lactulose for the treatment of hepatic encephalopathy. Dig Dis Sci. 2007;52(3):737-41

31. HCUPnet (Healthcare Cost and Utilization Project). Free health care statistics. 2018. Agency for Healthcare Research and Quality, Rockville, MD. Available at: https://hcupnet.ahrq.gov/\#setup. Accessed April 28, 2020.

32. Wai H, Stepanova M, Saab S, Erario M, Srishord M, Younossi ZM Inpatient economic and mortality assessment for liver transplantation: a nationwide study of the United States data from 2005 to 2009. Transplantation. 2014;97(1):98-103.

33. Bureau of Labor Statistics. Consumer Price Index-All Urban Consumers. 2018. Available at: http://data.bls.gov/cgi-bin/surveymost?cu. Accessed April 28, 2020

34. American Hospital Association. Table 4.4. Aggregate hospital paymentto-cost ratios for private payers, Medicare, and Medicaid, 1995-2016. In TrendWatch Chartbook 2018. Available at: https://www.aha.org/system/ files/2018-05/2018-chartbook-table-4-4.pdf. Accessed April 28, 2020.
35. Centers for Medicare $\&$ Medicaid Services. Cost to charge ratios. Tables 8A, 8B, 8C. FY 2019 Final Rule, Correction Notice, and Notice Tables. Available at: https://www.cms.gov/Medicare/Medicare-Fee-for-ServicePayment/AcuteInpatientPPS/FY2019-IPPS-Final-Rule-Home-Page-Items/ FY2019-IPPS-Final-Rule-Tables. Accessed May 14, 2020

36. Briggs AH, Weinstein MC, Fenwick EA, et al. Model parameter estimation and uncertainty: a report of the ISPOR-SMDM Modeling Good Research Practices Task Force-6. Value Health. 2012;15(6):835-42.

37. Grosse SD. Assessing cost-effectiveness in healthcare: history of the $\$ 50,000$ per QALY threshold. Expert Rev Pharmacoecon Outcomes Res. 2008;8(2):165-78

38. Sanyal A, Younossi ZM, Bass NM, et al. Randomised clinical trial: rifaximin improves health-related quality of life in cirrhotic patients with hepatic encephalopathy - a double-blind placebo-controlled study. Aliment Pharmacol Ther. 2011;34(8):853-61.

39. Bajaj JS, Pinkerton SD, Sanyal AJ, Heuman DM. Diagnosis and treatment of minimal hepatic encephalopathy to prevent motor vehicle accidents: a cost-effectiveness analysis. Hepatology. 2012;55(4):1164-71.

40. Huang E, Esrailian E, Spiegel BM. The cost-effectiveness and budget impact of competing therapies in hepatic encephalopathy - a decision analysis. Aliment Pharmacol Ther. 2007;26(8):1147-61.

41. Neff G, Zachry W 3rd. Systematic review of the economic burden of overt hepatic encephalopathy and pharmacoeconomic impact of rifaximin. Pharmacoeconomics. 2018;36(7):809-22.

42. Vlachogiannakos J, Viazis N, Vasianopoulou P, Vafiadis I, Karamanolis DG, Ladas SD. Long-term administration of rifaximin improves the prognosis of patients with decompensated alcoholic cirrhosis. J Gastroenterol Hepatol. 2013;28(3):450-55.

43. Bajaj J, Schubert C, Heuman D, et al. Persistence of cognitive impairment after resolution of overt hepatic encephalopathy. Gastroenterology. 2010;138(7):2332-40

44. Fu K, DiNorcia J, Sher L, et al. Predictive factors of neurological complications and one-month mortality after liver transplantation. Front Neurol. 2014;5:275.

45. Stracciari A, Guarino M. Neuropsychiatric complications of liver transplantation. Metab Brain Dis. 2001;16(1-2):3-11.

46. Wells CD, Murrill WB, Arguedas MR. Comparison of health-related quality of life preferences between physicians and cirrhotic patients: implications for cost-utility analyses in chronic liver disease. Dig Dis Sci. 2004:49(3):453-58. 